\title{
A COUNTABILITY CONDITION FOR PRIMARY GROUPS PRESENTED BY RELATIONS OF LENGTH TWO'
}

\author{
BY PAUL HILL
}

\author{
Communicated by Dana Scott, October 4, 1968
}

A subgroup $A$ of the $p$-primary group $G$ is nice if $p^{\alpha}(G / A)$ $=\left\{p^{\alpha} G, A\right\} / A$ for all ordinals $\alpha$. We consider the following countability condition: there exists a collection $\mathfrak{C}$ of nice subgroups of $G$ such that

(0) $0 \in \mathfrak{e}$.

(1) $\mathcal{C}$ is closed with respect to group-theoretic union in $G$.

(2) If $A \in \mathbb{C}$ and if $H$ is a subgroup of $G$ such that $\{A, H\} / A$ is countable, there exists $B \in \mathcal{C}$ such that $B \supseteq\{A, H\}$ and such that $B / A$ is countable.

The author [1] has referred to this condition as the third axiom of countability and has demonstrated that this is the countability condition-not the first axiom (countability) nor the second axiom (decomposition into a direct sum of countable groups)-which is truly relevant for the proof of Ulm's theorem.

In this note, we outline a short proof of

THEOREM. Suppose that $G$ is a p-primary group presented by an arbitrary number of generators $x_{i}(i \in I)$ and relations $R_{m}(m \in M)$. If each relation $R_{m}$ involves at most two generators, then $G$ satisfies the third axiom of countability.

PROOF. There is, of course, no loss of generality in assuming that the index sets $I$ and $M$ both contain an element denoted by 0 and that the relation $R_{0}$ is: $x_{0}=0$. By adding repeatedly, if necessary, new generators $y_{i}$ subject to defining relations of the form $p x_{i}=y_{i}$, we may assume that for each $i \neq 0$ in $I$ that there exists a relation $R_{m}$ of the form $p x_{i}=x_{j}$. Since each element of $G$ has order equal to a power of $p$, we may in fact assume that given any generator $x_{1} \neq x_{0}$ having order $p^{n}$ in $G$ that there exists a finite chain $x_{1}, x_{2}, x_{3}, \cdots, x_{n+1}=x_{0}$ of generators such that $p x_{i}=x_{i+1}$ is one of the given relations. Furthermore, by deleting certain redundancies in both generators and relations we may assume, in the end, that each relation $R_{m}, m \neq 0$, is precisely of the form $p x_{i}=x_{j}$ where $i \neq j$. For a quick verification of this, note that if

$$
r x_{i}=s x_{j} \quad \text { where } i \neq j \text { and }(r, p)=1
$$

\footnotetext{
1 The author acknowledges NSF support under Grant GP-8833.
} 
then $x_{i}=t x_{j}$ for some integer $t$ and that the generator $x_{i}$ is redundant for the presentation of $G$-with relations involving at most two generators. Having removed the redundancy (1) from the presentation of $G$, we observe that each relation $R_{m}, m \neq 0$, is implied by the given relations of the form $p x_{i}=x_{j}, i \neq j$, and $x_{0}=0$. Consider the relation

$$
R_{m}: r x_{i}=s x_{j},
$$

where $r$ and $s$ are integers. This relation is equivalent to, in the presence of the relations $p x_{i}=x_{j}$ and $x_{0}=0$, a relation of the form

$$
R_{m}^{\prime}: q x_{\lambda}=t x_{\mu} \quad \text { where }(q, p)=1 ;
$$

even if $r=0, r x_{i}=1 x_{0}$. The denial of (1) implies that $\lambda=\mu$, so the relation $R_{n}$ gives, beyond the information already given by the relations $p x_{i}=x_{j}$ and $x_{0}=0$, information only about the order of some generator $x_{\lambda}$. However, the order of each generator $x_{i}$ is already determined by the relations $p x_{i}=x_{j}$ and $x_{0}=0$.

We have shown that $G$ can be presented by generators $x_{i}(i \in I)$ and relations of the form $p x_{i}=x_{j}, i \neq j$, and $x_{0}=0$ such that

(i) if $i \neq 0$ is in $I$, there exists $j \in I$ such that $p x_{i}=x_{j}$ is a relation;

(ii) there is no redundancy of the form (1).

The proof of the theorem is clearly finished by the following lemma$G$ has plenty of nice subgroups.

Lemma. Suppose that the primary group $G$ is presented by generators $x_{i}(i \in I)$ and relations as described above. Let $J \subseteq I$ and let $H$ be the subgroup of $G$ generated by the generators $x_{j}, j \in J$. Then $H$ is a nice subgroup of $G$.

Proof. Define inductively for each ordinal $\alpha$ a subset $I_{\alpha}$ of $I$ in the following way: $I_{0}=I$,

and

$$
I_{\alpha+1}=\left[i \in I: p x_{j}=x_{i} \text { for some } j \in I_{\alpha}\right]
$$

$$
I_{\beta}=\bigcap_{\alpha<\beta} I_{\alpha} \text { if } \beta \text { is a limit ordinal. }
$$

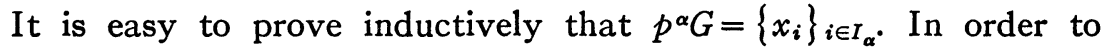
show that $H$ is a nice subgroup of $G$, we need to prove $p^{\alpha}(G / H)$ $=\left\{p^{\alpha} G, H\right\} / H$ for all $\alpha$. This, too, is an inductive argument and the nonlimit case is trivial. Suppose that $\beta$ is a limit and assume that $p^{\alpha}(G / H)=\left\{p^{\alpha} G, H\right\} / H$ for all $\alpha<\beta$. Let $x+H \in p^{\beta}(G / H)$. We may assume that $i \in J$ if $p x_{i}=x_{j}$ where $j \in J$. Write

$$
x=\sum_{j \in J} t_{j} x_{j}+\sum_{k \in K} t_{k} x_{k}, \quad \text { where }\left(t_{i}, p\right)=1
$$


and $K$ is disjoint from $J$. By the induction hypothesis, for each $\alpha<\beta$, there exists $h^{\alpha}=\sum_{j \in J} s_{j}^{\alpha} x_{j}$ in $H$ such that $x+h^{\alpha} \in p^{\alpha} G=\left\{x_{i}\right\}_{i \in I_{\alpha}}$. Thus

$$
\sum_{j \in J}\left(t_{j}+s_{j}^{\alpha}\right) x_{j}+\sum_{k \in K} t_{k} x_{k}=\sum_{i \in I_{\alpha}} r_{i} x_{i} .
$$

Since $\left(t_{k}, p\right)=1$ for each $k \in K$, it follows immediately from the limited substitution, $p x_{i}=x_{j}$, one can make on generators that $k \in I_{\alpha}$ for each $k \in K$. Hence $k \in I_{\beta}=\bigcap_{\alpha<\beta} I_{\alpha}$ for each $k \in K$. Defining $x^{\prime}=\sum_{k \in K} t_{k} x_{k}$, we have that $x^{\prime} \in p^{\beta} G$ and $x+H=x^{\prime}+H \in\left\{p^{\beta} G, H\right\} / H$. This verifies that $H$ is nice in $G$.

In connection with our result, the reader's attention is called to [2]; the connection should be obvious.

\section{REFERENCES}

1. Paul Hill, Ulm's theorem for totally projective groups, Notices Amer. Math. Soc. 14 (1967), 940.

2. Peter Crawley and Alfred Hales, The structure of torsion abelian groups given by presentations, Bull. Amer. Math. Soc. 74 (1968), 954-956.

Florida State University, Tallahassee, Florida 32306 